\title{
Microcontroller-based 9 Digits Code Lock System
}

\author{
Dr. Thida Aung \\ Professor and Head \\ Department of Electronic Engineering \\ Technological University (Lashio) \\ Lashio City, Northern Shan State, Myanmar
}

\author{
Dr. Kyaw Wuna \\ Director \\ Department of Academic Affairs \\ Technological University (Thanlyin) \\ Thanlyin Township, Yangon, Myanmar
}

\begin{abstract}
Security has been an important factor in life. We need homes, offices and our properties to be secured as much as possible to protect from unauthorized access. So security control systems and access control methods play vital role in modern technologies. A lot of research has been conducted on these methods and techniques. Discovering the more and more efficient control technology concerned with security becomes a challenging matter. In this paper, microcontroller-based code lock system which is simple in design, low in cost and high in level of security is presented. The system consists of microcontroller, 16x2 Liquid Crystal Display, 4x3 keypad and buzzer. The system has been developed and implemented by applying embedded control technique. The core value of the system is that it can provide user longer password length up to 9 digits compared with the other microcontroller-based password lock systems of which password length is just maximum 6 digits. So the system can provide higher level of security than any other system of its kind because longer password length makes the password more secure and the system more powerful in term of security. Moreover, the user can vary the password length as needed within the one to nine digits.
\end{abstract}

Keywords: Security; access control methods; microcontroller; embedded control technique; password length

\section{INTRODUCTION}

Due to many efforts and researches made in the field of security control techniques, today security control systems are very intelligent and give satisfactory results. But every system has its own advantages and disadvantages and no exception for these systems. The more advanced the system is, the more it costs and the more the system is complicated. In the environmental point of view, some systems operated in the very high frequency are hazardous to the health and pollute its associated environment. And some systems cause interference to nearby electronic devices. In this paper, 9 digits code lock system is presented to overcome these drawbacks. Although the code-protected security control system is not as secure as the biometric system like eye scanning, it is still critical in daily-life security-demanded applications especially in the protection of one's properties because of its cost-effectiveness and ease of use. Moreover it is user-friendly and does not pollute the environment. The proposed system is very simple in design and easy to implement. The block diagram of the 9 digits code lock system is shown in Figure 1. The power supply unit is for providing the required power for the whole system and power consumption is very low. Peripheral Interface Controller PIC16F877A produced by Microchip Technology is used as a main controller in this system. PIC microcontroller is selected because of its powerful features and its rich resources. Economically, it has low cost compared to other products. A four by three matrix keypad is used for entering the password. Keypad is the simplest form of electronic access control. Matrix keypad is a type of HumanMachine Interface (HMI) with numerical or function keys. A Liquid Crystal Display (LCD) is used for displaying the condition of the entered password and prompting for necessary information. In every step, LCD will show every instruction for the user and function of the microcontroller. Alarm unit is for alerting that an intruder or an illegitimate person is trying to gain access to the system. Buzzer is used for this purpose. Output signal will be activated when user enters the correct password. This means that user will gain access to the system.

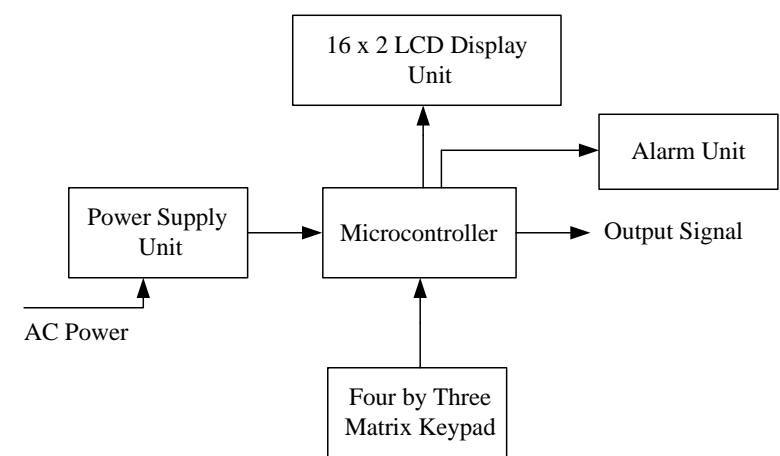

Figure 1. Block diagram of Microcontroller-based 9 Digits Code Lock System

\section{OPERATION OF THE SYSTEM}

When the system is powered, the LCD will display the text 'WELCOME TO YOU'. After that, the LCD will display the text 'SECURITY SYSTEM'. Then the microcontroller reads the previous stored password in its EEPROM location showing the message 'READING EEPROM' on the LCD. The microcontroller stores that read password in a specific RAM location. Then, the LCD will display the message 'ENTER PASSWORD'. At this time, the user can input the password by pressing the keys on keypad. While entering the password, the message 'SCANNING KEYPAD' will be displayed on the LCD. The entered password is stored into another RAM location. Two special characters ' $\#$ ' and ' $*$ ' are used. The key '\#' is used for 'ENTER' key and the key '*' is used for changing the password. The entered password is compared with the stored password. When the controller is comparing the passwords, the LCD will display the message 'COMPARING CODE'. If both the entered password is correct and the last character is '\#', the user will gain access to the system showing the message 'ACCESS GRANTED'. If the user inputs more than nine digits, only the last nine digits will be valid. Green LED is used in this system just for showing that the specific task is being executed. After five seconds time is up, the LED will turn off. If the entered 
password is incorrect, the user will be prohibited to access the system showing the message 'ACCESS DENIED'. The controller will give the user the next two chances to enter password. If the user enters incorrect password for three times, the controller will give an alarm notifying that the user is an illegitimate person. Alarm will continue until the power supply is cut-off. If the user wants to change password, the correct password together with the key ' $*$ ' must be entered. At that time, the LCD will display the message 'CHANGING CODE'. Then, the LCD will display 'ENTER PASSWORD'. The user must enter the new password and the special character '\#'. Then, the LCD will display the message 'CONFIRM AGAIN'. The user must input the new password again together with the special key '\#'. The controller will compare the next new password with the previous password. If they are not same, the password in EEPROM will not be changed showing the message 'CHANGING FAILED'. If they are same, the new password will be written into the EEPROM location showing the message 'CHANGING SUCCESS' The user must use the new password to access the system at the next time.

\section{SOFTWARE IMPLEMENTATION}

In developing the program, it must be sure that no programming or logical errors present in the program as the whole system totally depends on the program. In this system, MPLAB Integrated Development Environment (IDE) software developed by Microchip Technology is used for developing the program. Assembly language, one of the high level programming languages, is used as the programming language. Utilizing the rich resources of PIC, EEPROM data reading and writing techniques, indirect memory accessing technique and software debouncing technique are applied in this system. The main program is composed of many subroutines or procedures. Each procedure is created for specific functions or tasks. Critical subroutines contained in this main program are explained in detail with respective flowcharts. Figure 2 shows the main flowchart for code lock system.

\subsection{Scan Procedure}

It is used for scanning the user's input and saving this in the buffer, and passing the data concerned with the input to the respective section of the program. Keyscan procedure for sensing which key is entered is nested in this procedure. Readchk_enter subroutine, a nested subroutine of scan procedure, is for checking whether the special keys, '*' for code change and '\#' for enter, are pressed or not. The scan procedure also makes the last nine digits of the entered password valid if the user enters more than nine digits because the system password length is only nine digits. To read keys pressed, the program requires the switch debouncing. This means that the delay of approximately 20 to 30 milliseconds is needed between button presses. This part is required to allow the key hits to settle for a few tens of milliseconds before establishing a firm contact. In this program, 24 milliseconds delay is used for debouncing. The flowchart for scan procedure is shown in Figure 3.

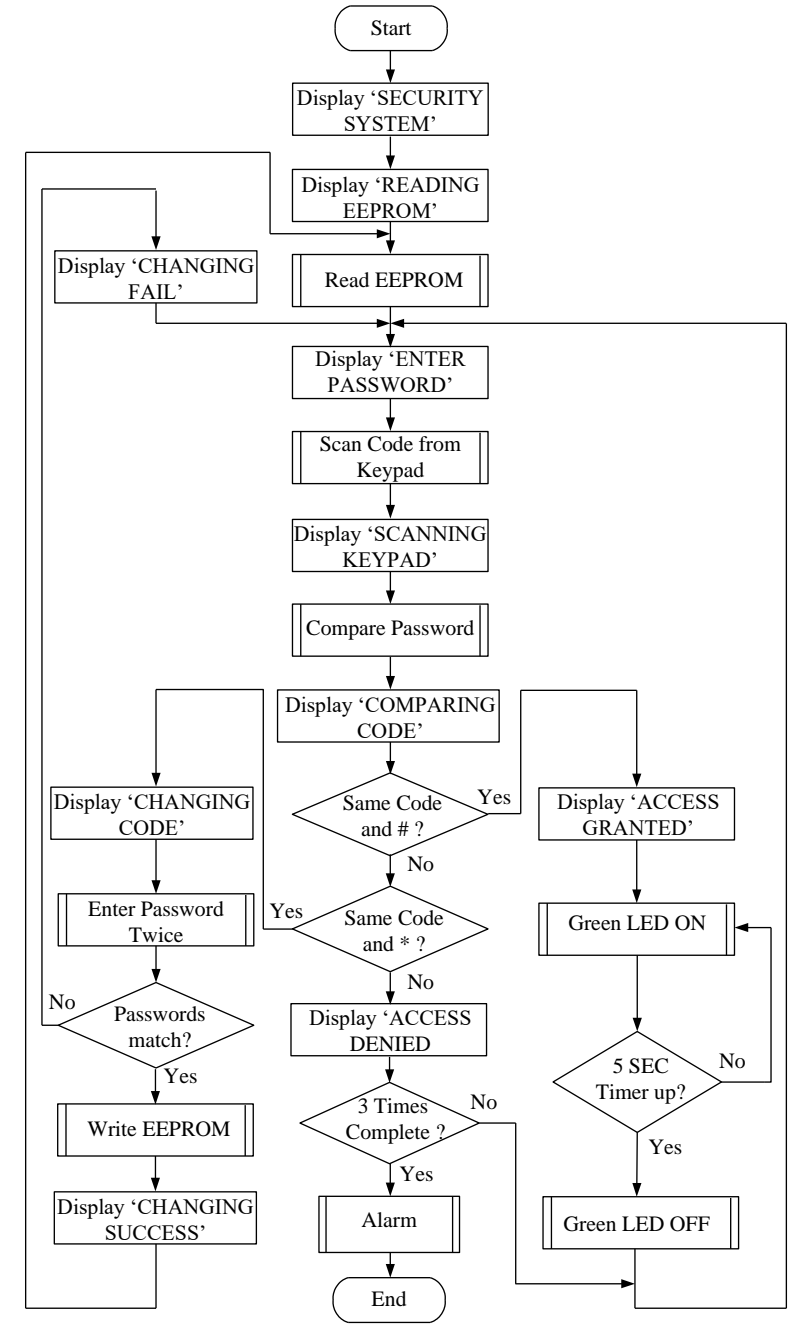

Figure 1. Flowchart for the overall system

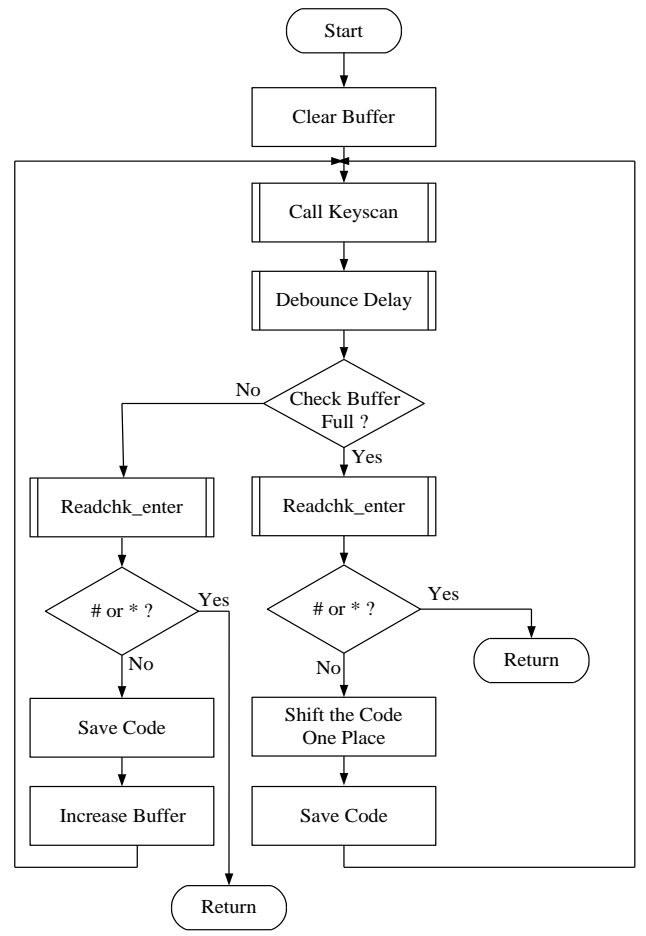

Figure 3. Flowchart for scan procedure 


\subsection{Readchk_enter Procedure}

This procedure checks the keycode from RAM register locations whether the last character is the same as '\#' key or '*' key. If it is '\#' key or '*' key, it will return with zero flag bit of status register, $\mathrm{Z}=1$. Otherwise it will return with $\mathrm{Z}=$ 0 . The flowchart for this procedure is shown in Figure 4.

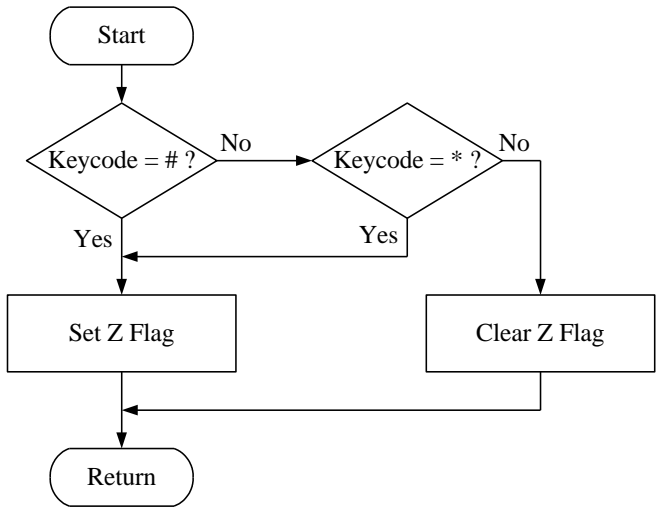

Figure 4. Flowchart for readchk_enter procedure

\subsection{Compbuf Procedure}

Compbuf procedure is created to compare the code from RAM locations with the entered code in read buffer. In other words, this procedure checks whether the user's entered codes and the stored codes in the microcontroller. When the user wants to change the password, it checks the confirmation password whether they are same or not. If they are same, it will return with $Z=1$, otherwise $Z=0$. The flowchart for compbuf procedure is shown in Figure 5.

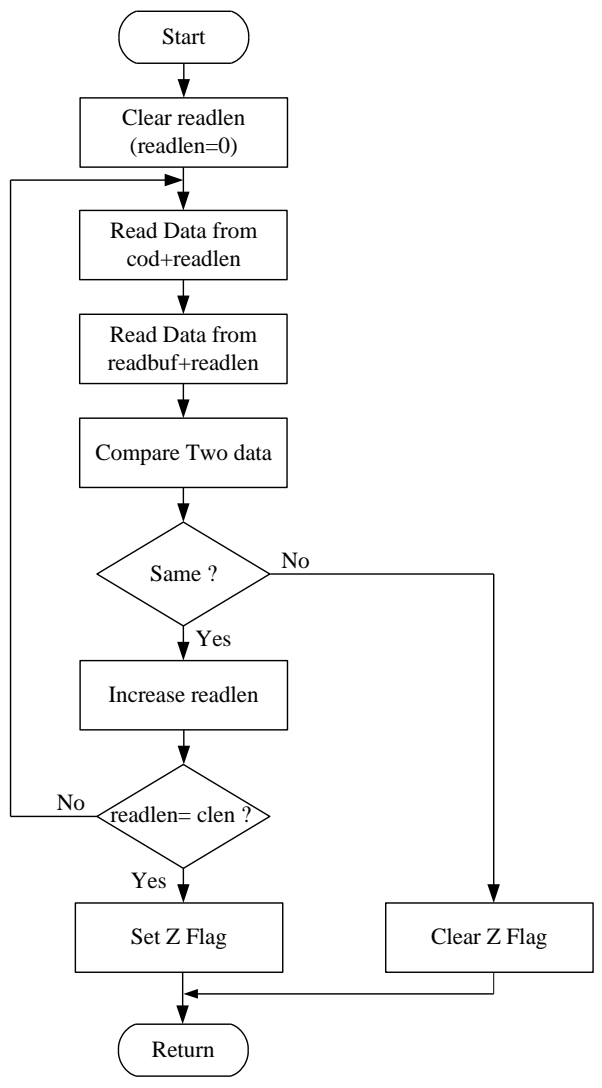

Figure 5. Flowchart for combuf procedure

\section{HARDWARE IMPLEMENTATION}

By utilizing the efficient resources of the PIC, the circuit of the whole system is very compact and very simple. If power supply is not taken into account, all necessary components in this circuit are just only keypad, LCD, $4 \mathrm{MHz}$ crystal oscillator, LED, buzzer, a general purpose transistor, two ceramic capacitors and some resistors. The entire circuit diagram is shown in Figure 8. The main components of the system will be explained briefly.

\subsection{Microcontroller}

PIC 16F877A is chosen as main controller for this system because it meets the requirements of the system and it has rich of features. PIC 16F877A is the mid-range pic and it has 40 pins.

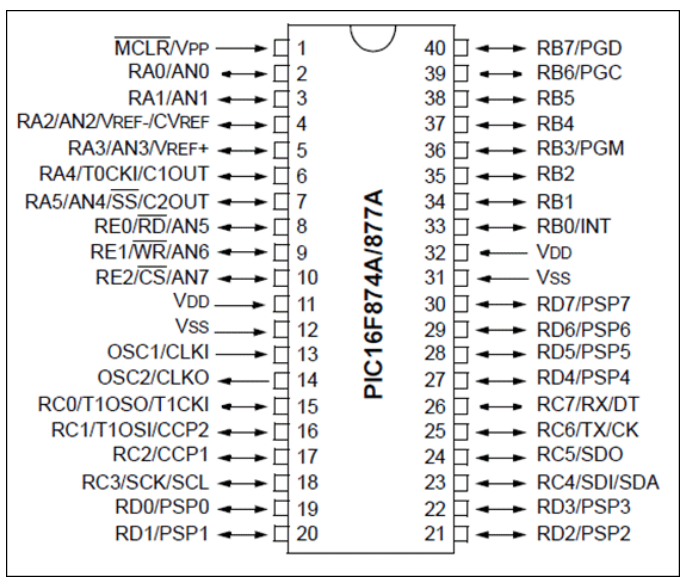

Figure 6. Pin diagram of PIC 16F877A

\subsection{6x2 LCD Display}

LCD (Liquid Crystal Display) screen is an electronic display module and find a wide range of applications. A 16x2 LCD display is very basic module and is very commonly used in various devices and circuits. These modules are preferred over seven segments and other multi segment LEDs. The reasons being: LCDs are economical; easily programmable; have no limitation of displaying special \& even custom characters (unlike in seven segments), animations and so on. A 16x2 LCD means it can display 16 characters per line and there are 2 such lines.

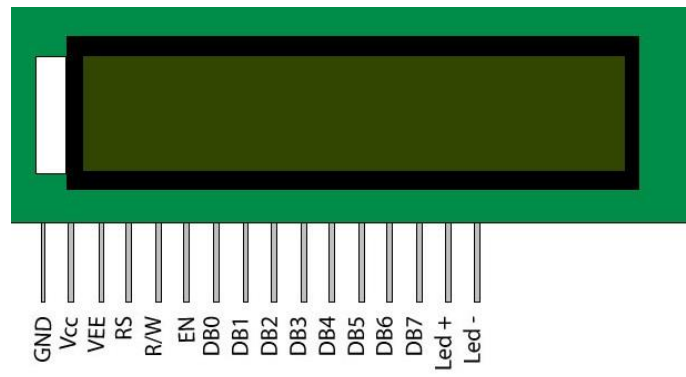

Figure 7. Pin diagram of $16 \times 2$ LCD Display

\subsection{4x3 Matrix Keypad}

The keypad is a part of the input device used in this system to input authentication digits. Keypads are organized in a matrix of rows and columns. When a key is pressed, a row and a column make a contact; otherwise, there is no connection between rows and columns. A 4x3 matrix keypad means 4 rows and 3 columns keypad with total 12 combination patterns. 


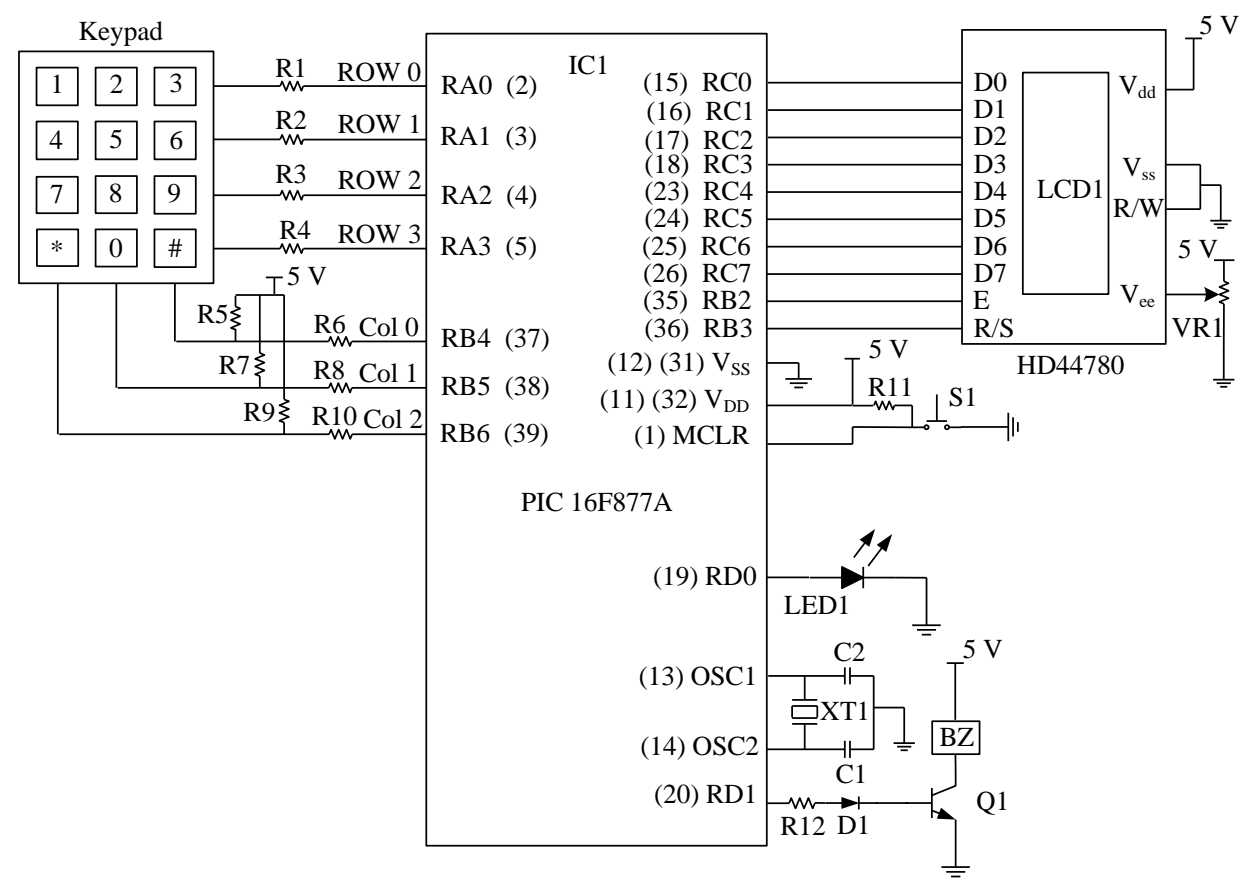

Figure 8. Schematic circuit diagram of the code lock system

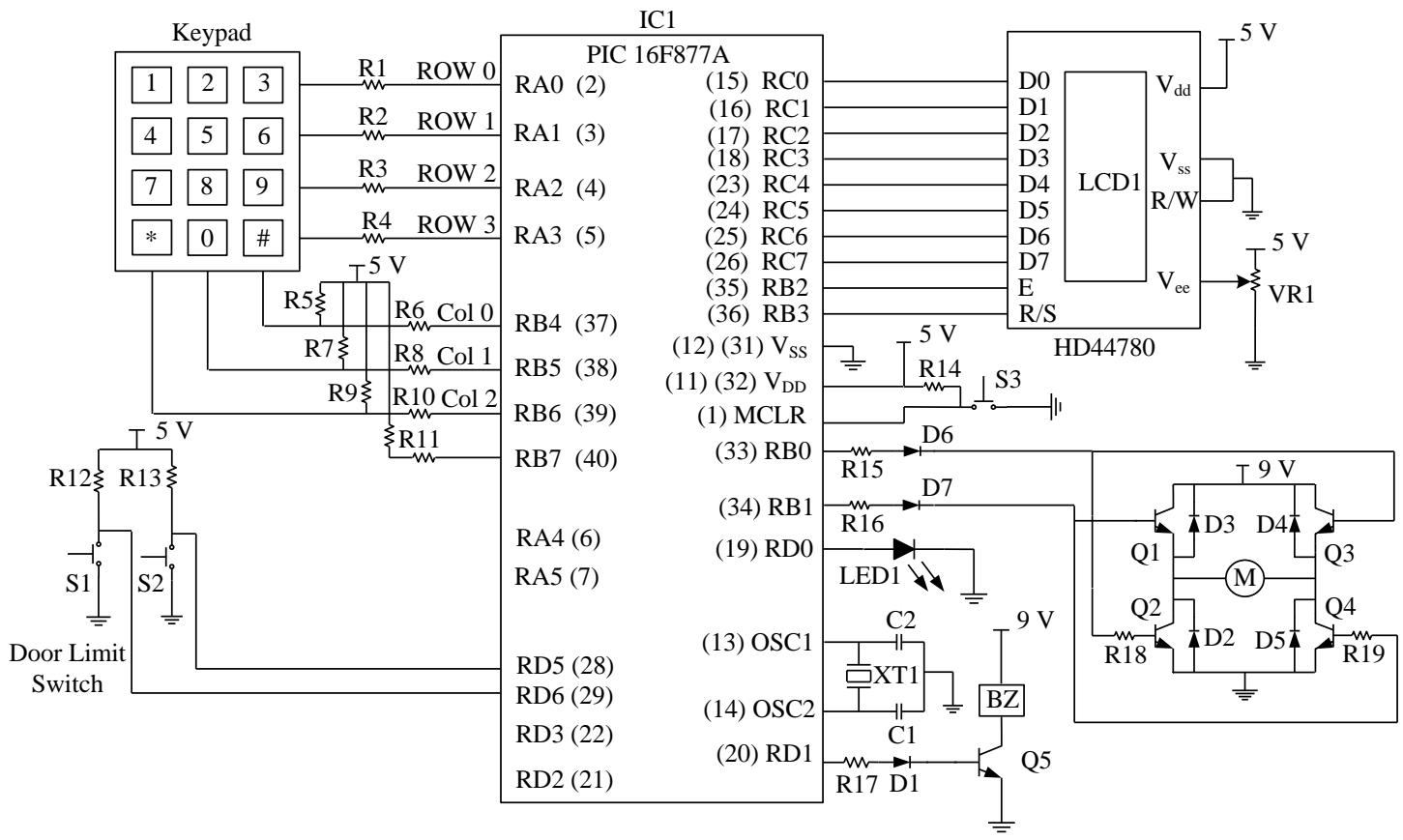

Figure 9. Schematic circuit diagram of the code-protected door control system

\section{DOOR CONTROL SYSTEM}

Door control system is presented here just for an example as one of the applications of the proposed system and for showing the idea how the proposed system can be applied for the desired application areas. Just modifying the code lock system a little, code-protected door control system can be implemented. In this system, door will be opened if the password is correct and automatically closed after a reasonable time delay. Time delay is set to 10 seconds. The alarm will be activated if the password is entered incorrectly for three times. Schematic circuit diagram of the codeprotected door control system is shown in Figure 9.
6. SIMULATION, TESTING \& RESULTS

Simulation is necessary for monitoring and evaluating the expected results. In this paper, simulation is performed by using ISIS software, the product of Labcenter Electronics. It is a very powerful tool for developing the embedded system designs. This product combines mixed mode circuit simulation, micro-processor models and interactive component models to allow the simulation of complete microcontroller based designs. Simulation of the code lock system is shown in Figure 10 and simulation of the door control system is shown in Figure 11. Experimental testing of the two systems is shown in Figure 12 and Figure 13 respectively. 
cX

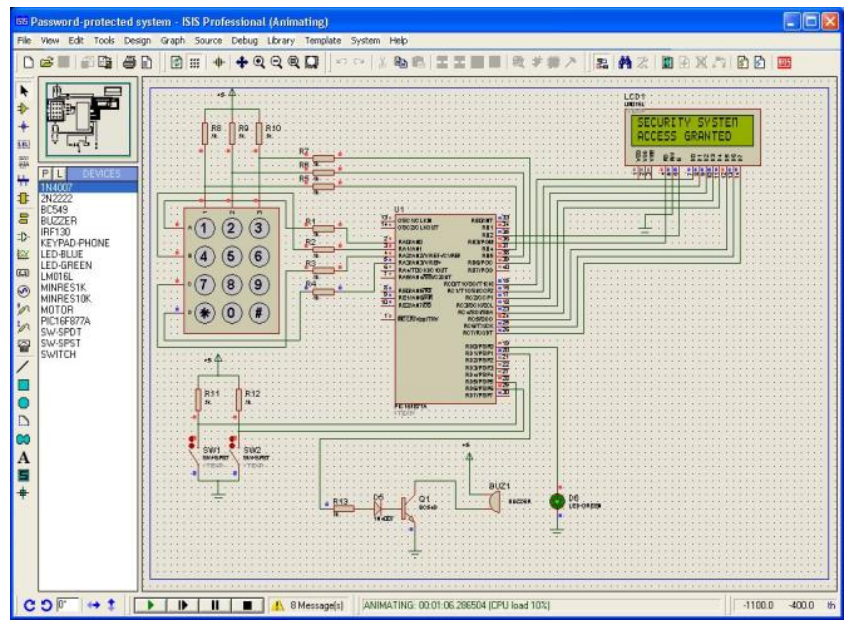

Figure 10. Simulation of the code locks system

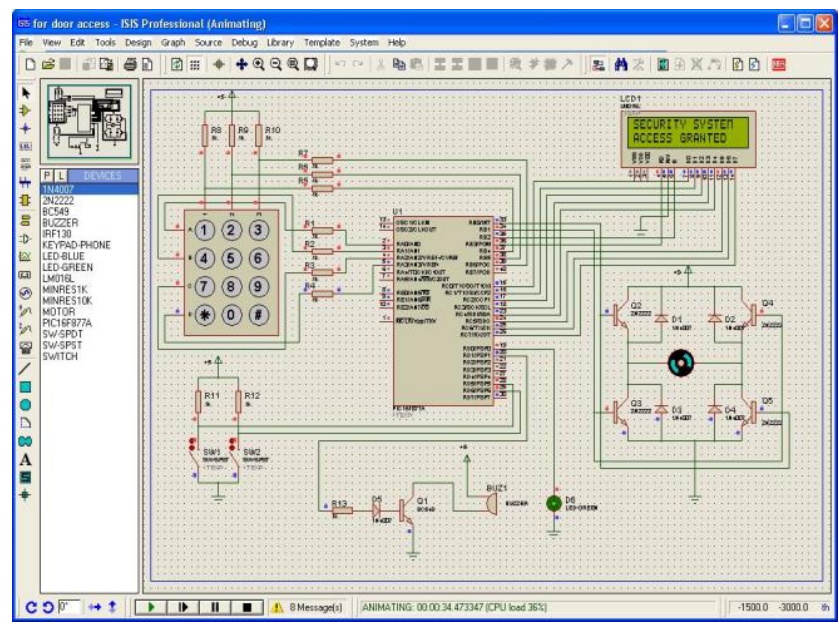

Figure 11. Simulation of the code-protected door control system

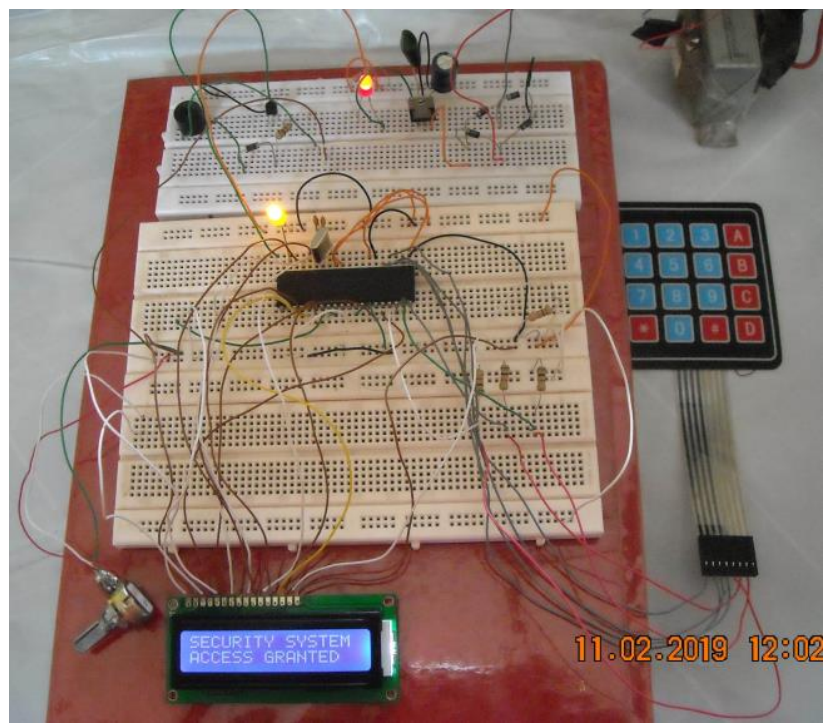

Figure 12. Experimental testing of the code lock system

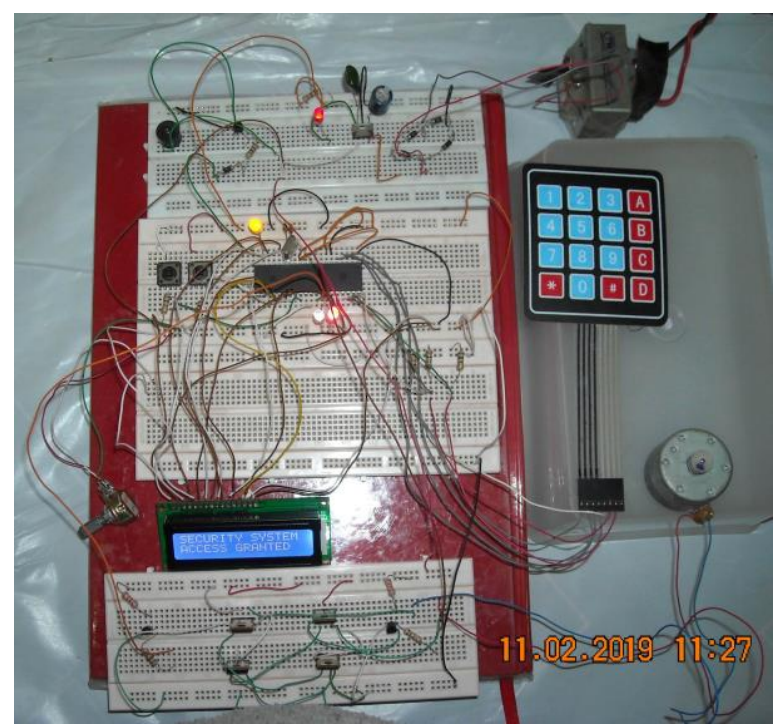

Figure 13. Experimental testing of the code-protected door control system

\section{CONCLUSION}

In this paper, microcontroller-based 9 digits code lock system has been presented. By taking advantages of the rich resources and features of PIC, the required program for the system has been developed. EEPROM data reading and writing techniques, indirect memory accessing technique and software debouncing technique are applied in developing the program. Assembly programming language has been used for developing the program. The developed program together with the system circuit has been simulated by ISIS interactive simulation software. As this software supports the real-time interactive simulation, the necessary changes in software and hardware can be made even in the developing phase. The circuit of the implemented system is very compact and straightforward as the system is based on the embedded system design.

\section{ACKNOWLEDGMENTS}

The authors would like to express their sincere gratitude and deep appreciation to Dr. Theingi, Rector, Technological University (Thanlyin), because of her valuable suggestions and guidance. The author also thanks to my colleagues in Department of Electronic Engineering, Technological University (Lashio) for their help during developing this system.

\section{REFERENCES}

[1] M. James, 2001. PIC and 8051 Microcontroller Cook Book, 2nd ed., Biddles Ltd.

[2] M. Predko, 2000. Programming and Customizing PIC Microcontrollers, 2nd ed., McGraw-Hill Inc.

[3] B. J. Peakman, 2000. Design with PIC Microcontrollers, 2nd ed., Prentic-Hall International Inc.

[4] J. Iovine, 2000. PIC Microcontroller Project Books, McGraw-Hill Inc.

[5] PIC 16F877A Datasheet, Microchip Technology Inc. http://ww1.microchip.com/downloads/en/DeviceDoc/ 39582C.pdf

[6] D. W. Smith, 2002. PIC in Practice, Gutenberg Press Ltd. 